\title{
Steady-frequency waves at intradiurnal periods from simultaneous co-located microbarometer and seismometer measurements: a case study
}

\author{
G. M. Shved, N. V. Karpova, L. N. Petrova, E. G. Orlov, and S. I. Ermolenko \\ Departments of Atmospheric Physics and Earth Physics, St. Petersburg State University, St. Petersburg-Petrodvorets 198504, \\ Russia
}

Received: 29 November 2010 - Revised: 19 May 2011 - Accepted: 30 May 2011 - Published: 29 June 2011

\begin{abstract}
Simultaneous co-located microbarometer and vertical-pendulum seismometer measurements at St. Petersburg $\left(59.9^{\circ} \mathrm{N}, 29.8^{\circ} \mathrm{E}\right)$ with total duration of 4 months are used to study atmospheric oscillations at steady frequencies at periods shorter than $8 \mathrm{~h}$. The temporal behavior of the phase shift between oscillations detected simultaneously by both instruments is analyzed for oscillations of periods up to as short as $\sim 0.5 \mathrm{~h}$. Some of oscillations last up to several days. For the $42-90 \mathrm{~min}$ and $2.5-5 \mathrm{~h}$ period ranges, the temporal behavior of the power spectra are considered and correlated with the atmospheric angular momentum of zonal wind, tropical cyclones and large earthquakes. Signatures of sequences of subharmonics of the solar tide are revealed with periods up to day/30. There are indications of effects of the 5-day planetary waves and tropical cyclones on wave activity in the $\sim 1-5 \mathrm{~h}$ period range. A weak increase of wave activity is observed when large earthquakes occur.
\end{abstract}

Keywords. Meteorology and atmospheric dynamics (Middle atmosphere dynamics; Waves and tides)

\section{Introduction}

The investigation is concerned with atmospheric waves at steady frequencies in the $\sim 0.5-8 \mathrm{~h}$ period range. These waves include subharmonics of the solar tide and atmospheric normal modes. The study is based on simultaneous co-located microbarometer and seismometer measurements of pressure and gravity, respectively, both at the surface. Observed gravity perturbations with time scales more than $0.5 \mathrm{~h}$ are mainly caused by atmospheric motions since these result in a continuous redistribution of air masses and accordingly the gravity varies due to the Newtonian attraction by

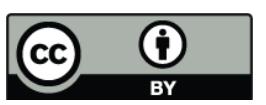

Correspondence to: G. M. Shved (shved@ pobox.spbu.ru) these masses (e.g., Farrell, 1972; Spratt, 1982; Müller and Zürn, 1983; Merriam, 1992; Crossley et al., 1995; Zürn and Widmer, 1995; Boy et al., 1998). Up to now, seismometer measurements are not exhaustively used for studying the above-mentioned short-period global waves. The purpose of this paper is to attract attention to these measurements as a promising method for wave investigations.

Using simultaneous microbarometer and superconducting gravimeter measurements with the duration no less than one year, Warburton and Goodkind (1977), Smylie et al. (1993), and Crossley et al. (1995) have detected subharmonics of the solar tide, $\mathrm{S}_{m}$ ( $m$ is the number of cycles per day), up to $m \sim$ 10 (corresponding period is $\sim 2.5 \mathrm{~h}$ ) in the variations of both pressure and gravity at different midlatitude sites in Europe and Northern America. The $S_{m}$ subharmonics up to $m=10$ have also been revealed by Livneh et al. (2007) from a 1-year record of surface-pressure variation at the low-latitude site Arecibo. The 5-years of measurements by a superconducting gravimeter at Strasbourg have even revealed $\mathrm{S}_{m}$ subharmonics up to $m=23$ (corresponding period is $\sim 1 \mathrm{~h}$ ) (Florsch et al., 1995). High $S_{m}$ subharmonics up to $m=8$ has been obtained by Walterscheid and Sivjee $(1996,2001)$ from winter observations of mesopause $\mathrm{OH}$ Meinel airglow in the Northern and Southern polar caps. One may think (Sect. 5) that Gerrard et al. (2010) have revealed higher $S_{m}$ subharmonics from measurements of the 630.0-nm OI and 427.8-nm N $\mathrm{n}_{2}^{+}$ thermospheric emission at two sites in the Antarctic during three austral winters. The high-latitudinal high $\mathrm{S}_{m}$ subharmonics have been explained as zonally-symmetric nonmigrating tides with maximum amplitudes for temperature and density at the poles (Walterscheid and Sivjee, 1996).

Theory predicts the existence of atmospheric normal modes in the $\sim 0.5-8 \mathrm{~h}$ period range (e.g., Dikii, 1965). Their frequencies depend on the spatial distributions of background wind and temperature (e.g., Forbes, 1995), which change strongly with the seasonal cycle. The variability of normal mode frequencies makes it difficult to detect the weak

Published by Copernicus Publications on behalf of the European Geosciences Union. 


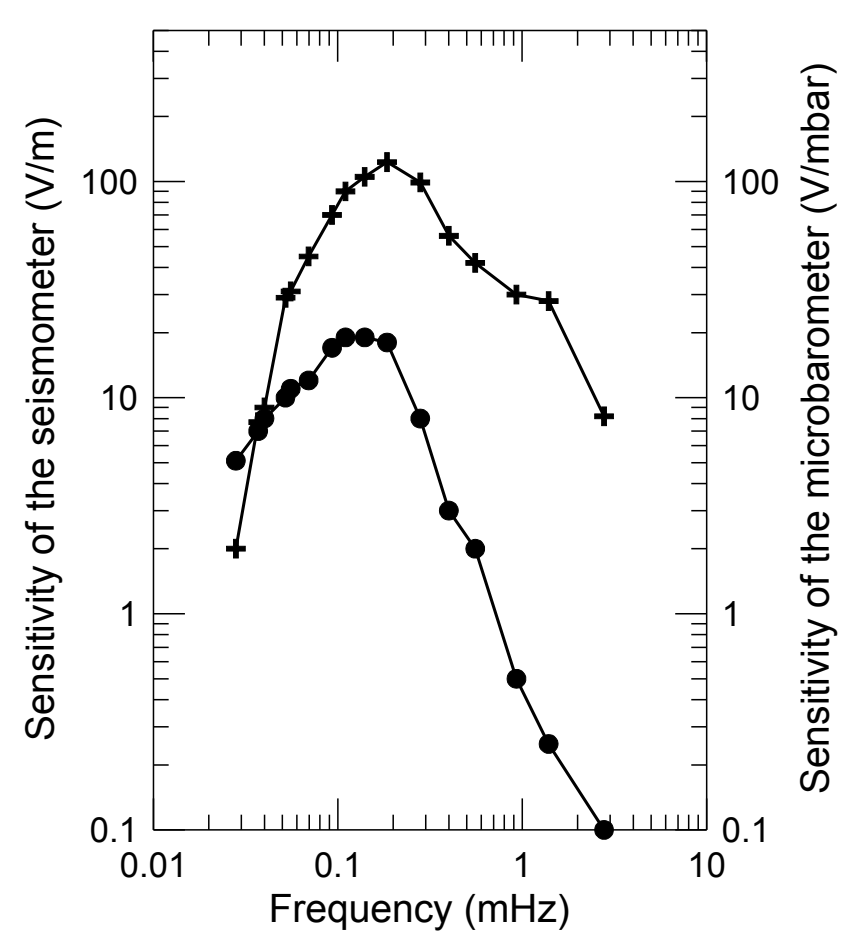

Fig. 1. Amplitude-frequency responses of microbarometer (circles) and seismometer (crosses).

modes at the considered periods by spectral analysis of long time series (with the length of 1 year and longer). The 11.5-h mode is the mode of the shortest period, which has been detected by Hamilton and Garcia (1986) from multi-year surface-pressure measurements at tropical stations. However, the meteor radar wind observations near the mesopause (e.g., Forbes et al., 1999b) and satellite measurements of thermospheric density near $200 \mathrm{~km}$ (Forbes et al., 1999a) have revealed normal modes up to periods of $\sim 7$ and $\sim 6 \mathrm{~h}$, respectively.

Since both the high $\mathrm{S}_{m}$ subharmonics and the normal modes are expected to be transient waves, Karpova et al. $(2002,2004)$ have used the technique of multiplication of power spectra for short time series. They have applied this technique to the simultaneous microbarometer and verticalpendulum seismometer measurements at St. Petersburg, simultaneous identical measurements at Schiltach, Germany, and vertical-pendulum seismometer measurements at Collm, Germany. The distance of Collm and St. Petersburg from Schiltach is about 500 and $2000 \mathrm{~km}$, respectively. The total duration of the measurements at each site was no less than 16 months. The long records were divided into the 2-3.5 day segments. The product spectra analyzed were obtained by multiplying the power spectra for these segments separately for each site and instrument. Both the microbarometer and seismometer measurements at all the sites have shown that steady-frequency oscillations in the $\sim 1-5 \mathrm{~h}$ period range exist at any site and in each season. Common oscillations have been detected in pressure and gravity variations as well as revealed at different sites. Some of the oscillations are $S_{m}$ subharmonics with $m$ up to $\sim 30$. Moreover, the $8-\mu \mathrm{Hz}$ periodicity signals are revealed in the frequency distribution of the oscillations. This periodicity can be interpreted as a signature of the gravitational normal modes since the asymptotic formula for the frequencies of these modes in the limit of small periods (Dikii, 1965) predicts the $\sim 8-\mu \mathrm{Hz}$ spacing between adjacent modes. Earlier, Petrova and Shved (2000) have detected this spacing between peaks in the gravity spectrum obtained from four vertical-pendulum seismometer measurements at St. Petersburg with a total duration of 9 days.

In this study, we analyze simultaneous co-located measurements of surface pressure and gravity variations in autumn with a total duration of 4 months. We consider temporal changes in the power spectra of pressure and gravity variations, obtained individually for high- and low-frequency subranges of the $0.7-5 \mathrm{~h}$ period range. The number of spectral peaks in the spectra derived for consecutive time samples of records is nontraditionally taken as a measure of wave perturbation of pressure and gravity to find effects of the dynamic and thermal state of the atmosphere on the spectra. The axial components of the atmospheric angular momentum associated with zonal wind individually in the Northern and Southern Hemispheres are adopted as indicators of the atmospheric state on a global scale. Influences of both the tropical cyclones and large earthquakes on the wave perturbations are examined. Spectra of coherence between microbarometer and vertical-pendulum seismometer records for the $\sim 0.5$ $12 \mathrm{~h}$ period range are also calculated. The temporal behavior of phase shift between the oscillations of pressure and gravity is determined at the frequencies of statistically reliable peaks in these spectra.

\section{Instrumentation and input data}

The microbarometer used has been derived from the standard millibarometer M-22 (Kozhevnikova et al., 1980). The seismometer with Kirnos vertical pendulum (Savarensky and Kirnos, 1955) is enclosed in a sealed chamber to protect it from a direct effect of variations in ambient air pressure and temperature (Lin'kov, 1987). The photoelectric transducer has been constructed in such a way that a feedback can compensate the zero drift of the seismometer. Moreover, a heat insulation of the chamber slows down the drift. As a result, the zero point correction has to be performed only once in several months. Both instruments are adapted to the measurements of the $\sim 1-5 \mathrm{~h}$ period oscillations (Fig. 1). The peak sensitivity of the microbarometer and seismome-

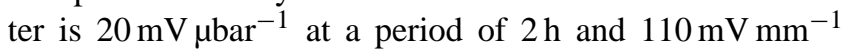
at a period of $1.5 \mathrm{~h}$, respectively. This yields a resolution (at these periods) equal to $\sim 0.1 \mu$ bar for pressure variations and $\sim 20 \mu \mathrm{m}$ for surface displacements or $\sim 2 \mathrm{nGal}$ for 

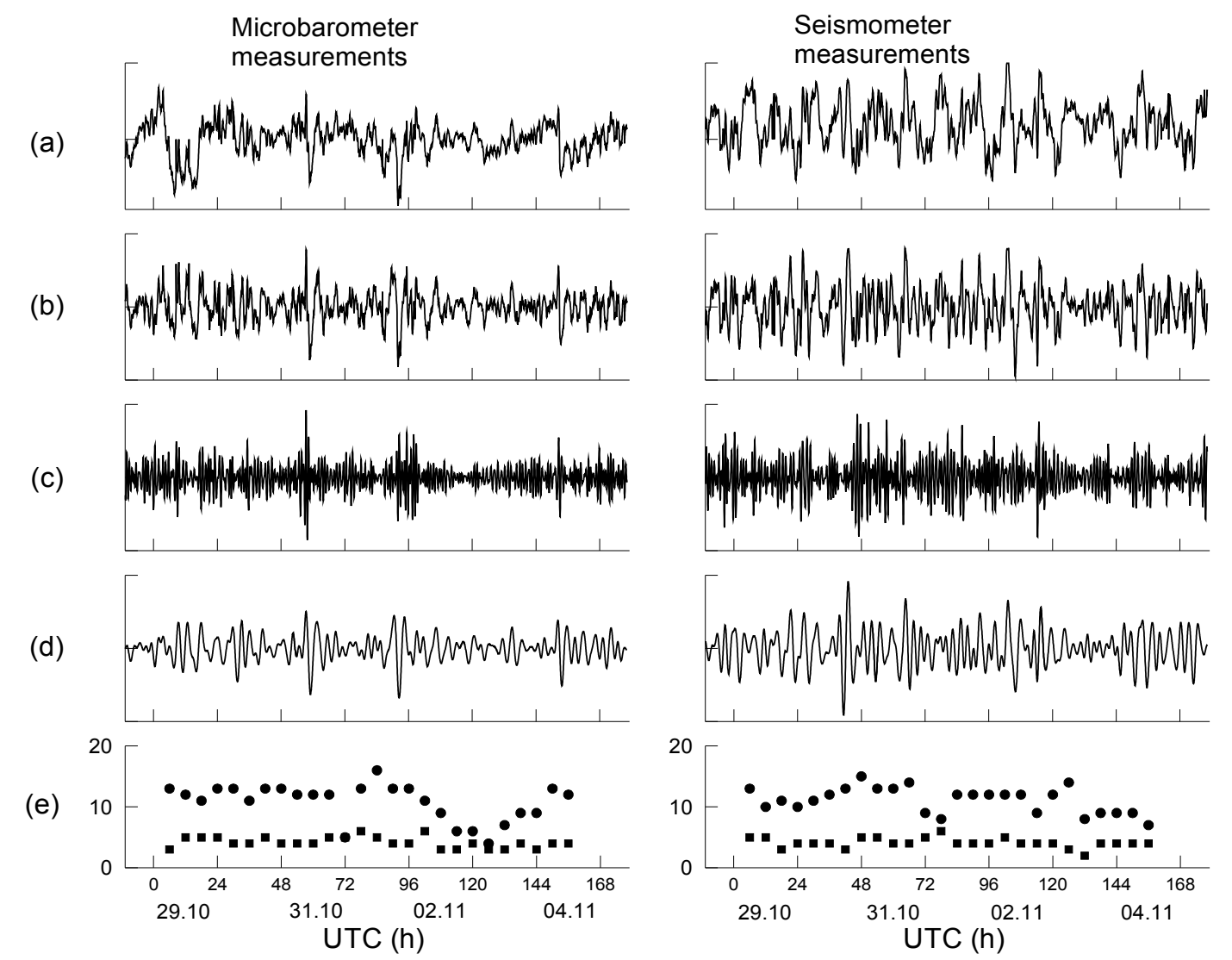

Fig. 2. Raw records of simultaneous microbarometer and seismometer measurements for 29 October-4 November 1997 (a). These records after removing the smoothed curve obtained with the sliding 12-h window (b) and a subsequent application of the bandwidth Potter filters for separating the "high-frequency" oscillations in the 42-90 min period range (c) and the "low-frequency" oscillations in the 2.5-5 $\mathrm{h}$ period range (d). All the series are given in relative units. The numbers of peaks in the spectra of 35-h segments of the records (e) for the "high-frequency" (circles) and "low-frequency" (squares) subranges.

acceleration $\left(1 \mathrm{Gal}=1 \mathrm{~cm} \mathrm{~s}^{-2}\right)$. The sampling time of the measurements is $1 \mathrm{~min}$.

The simultaneous microbarometer and seismometer measurements have been performed at St. Petersburg $\left(59.9^{\circ} \mathrm{N}\right.$, $29.8^{\circ} \mathrm{E}$ ). We have analyzed autumn records from 30 August to 3 November 1996 and from 29 August to 4 November 1997. These records also have previously been used by Karpova et al. $(2002,2004)$. Because of the technical features of the recorder, each continuous record for both the instruments is limited to week in length. An example of the records is shown in Fig. 2a.

\section{Temporal behavior of oscillation spectra}

\subsection{Procedure of record analysis}

Each of continuous records was processed individually. To eliminate long-term trends, the sliding 12-h window was applied to raw records (an example is given in Fig. 2b). Then, a Potter bandwidth filter was applied to obtain time series in- dividually for the consideration of "high-frequency" oscillations in the $185.15-396.5 \mu \mathrm{Hz}$ subrange (42-90 min periods) and "low-frequency" oscillations in the $55.55-110.72 \mu \mathrm{Hz}$ subrange (2.5-5 h periods) (see Fig. $2 \mathrm{c}$ and d).

Power spectra were calculated for short segments that had been selected from the filtered record. On the one hand, the length of these segments should be reasonably small to assume the steadiness of the waves examined. On the other hand, it should be sufficiently large to provide a frequency resolution no worse than $8 \mu \mathrm{Hz}$ for revealing the atmospheric normal modes. A length of $35 \mathrm{~h}$ is thus a compromise between these contradictory requirements. The ends of short series were smoothed with a cosinusoidal function.

Fast Fourier transformation was applied over the 35-h windows sliding along the filtered records with 15-min step. The set of consecutive power spectra for each continuous record then presents a dynamic spectrum. Examples are shown in Figs. 3 and 4. As a result, for the 4 months of measurements we have obtained the 21 dynamic spectra of 5.5 days length on the average for both the instruments in each frequency subrange. 


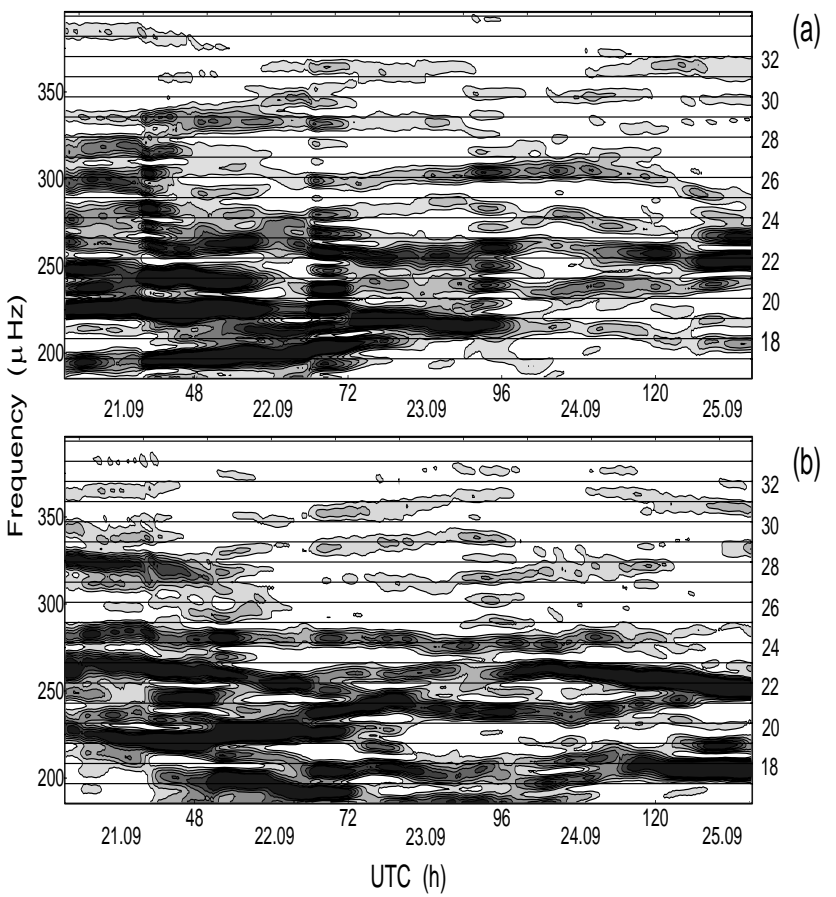

Fig. 3. Temporal behavior of power frequency spectra (in arbitrary units) in the 42-90 min period range, derived from microbarometer (a) and seismometer (b) measurements during 21-25 September 1997. Blacking indicates spectral intensity more than one third of the maximum magnitude, contour interval is $1 / 18$ of this magnitude. The time is counted from the beginning of the record. The horizontal lines correspond to the frequencies of solar tide subharmonics. The numbers at these lines indicate subharmonic numbers.

As mentioned in the Introduction, we are interested here in steady-frequency oscillations. However, it cannot be ruled out that our power spectra contain transient peaks resulted mainly from atmospheric internal gravity waves (IGW). Since IGWs are generated by short-lived local perturbations in the atmosphere, these waves are also short-lived and expected to result in weak spectral peaks (see about IGW in Sect. 5 in more details). Then, as a measure of atmospheric perturbations forced by steady-frequency oscillations we use the number $N$ of peaks in a spectrum, which have one of two following features. First, peaks which exceed one third of height of the highest peak in a considered spectrum are taken. Second, of the rest we take peaks which correspond to oscillations lasting more than $12 \mathrm{~h}$ with almost constant frequencies. The $N$ numbers have been individually counted for each instrument in each frequency subrange at 00:00, 06:00, 12:00, and 18:00 UTC. An example of the series of the $N$ numbers is given in Fig. 2e. The implications of analysis with the use of $N$ numbers are justified if they are reproduced from different data and can be reasonably explained by theory.

The reliability of oscillations revealed in the power spectra was tested by using the Lomb-Scargle spectral analysis

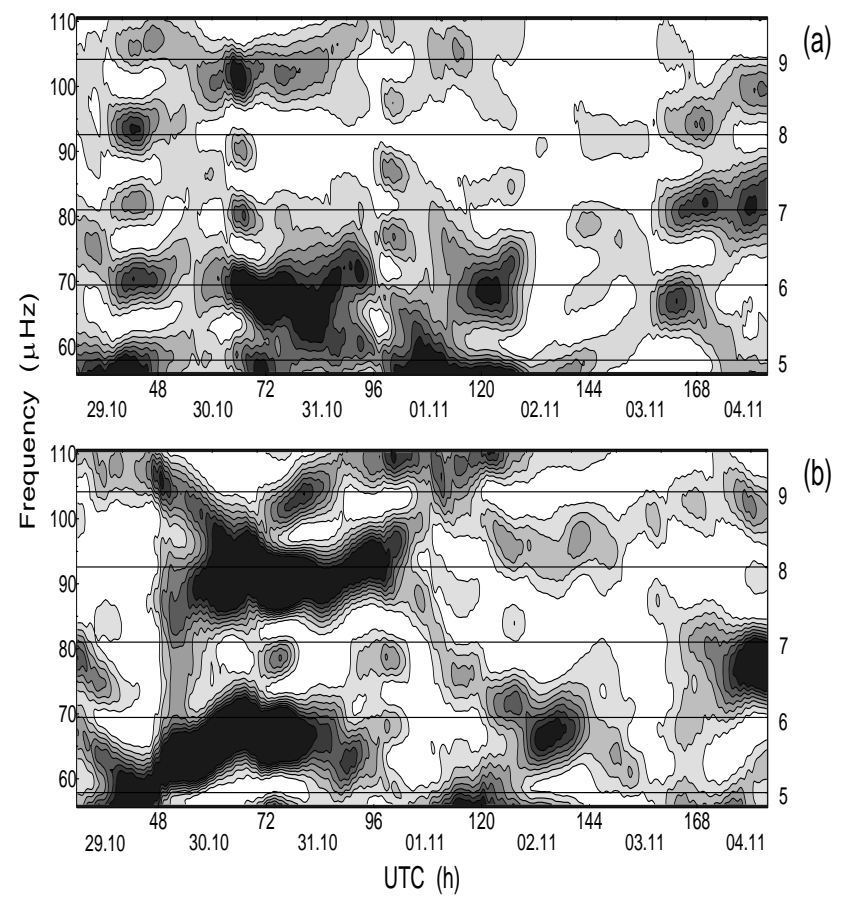

Fig. 4. As for Fig. 3 but in the $2.5-5 \mathrm{~h}$ period range during 29 October-4 November 1997.

(Press et al., 1992) which results in the periodogram power normalized by the total variance of the data. The statistical significance of spectral peaks was estimated by comparing their normalized observed powers $z$ with normalized periodogram power for the white (Gaussian) noize, which has the $\exp (-z)$ probability distribution (Scargle, 1982). Examples of verifying the reliability of revealed oscillations in the low- and high-frequency subranges are respectively shown in Fig. 5 by processing the raw records and Fig. 6 by processing the filtered series. Figure 5 also shows that the commonly observed solar and lunar tides contribute to the data analyzed here.

\subsection{Special features of spectra}

Results from the analysis of dynamic spectra are the same for both the microbarometer and seismometer measurements:

1. In both frequency subranges there are oscillations with almost constant frequencies, which last up to several days (see Figs. 3 and 4).

2. In all the "low-frequency" dynamic spectra and in most of the "high-frequency" ones there are sets of oscillations separated approximately by the $11.6 \mu \mathrm{Hz}$ interval equal to the spacing between adjacent $\mathrm{S}_{m}$ subharmonics.

3. The frequency of some oscillations varies strongly in time (up to $\sim 30 \mu \mathrm{Hz}$ ). 

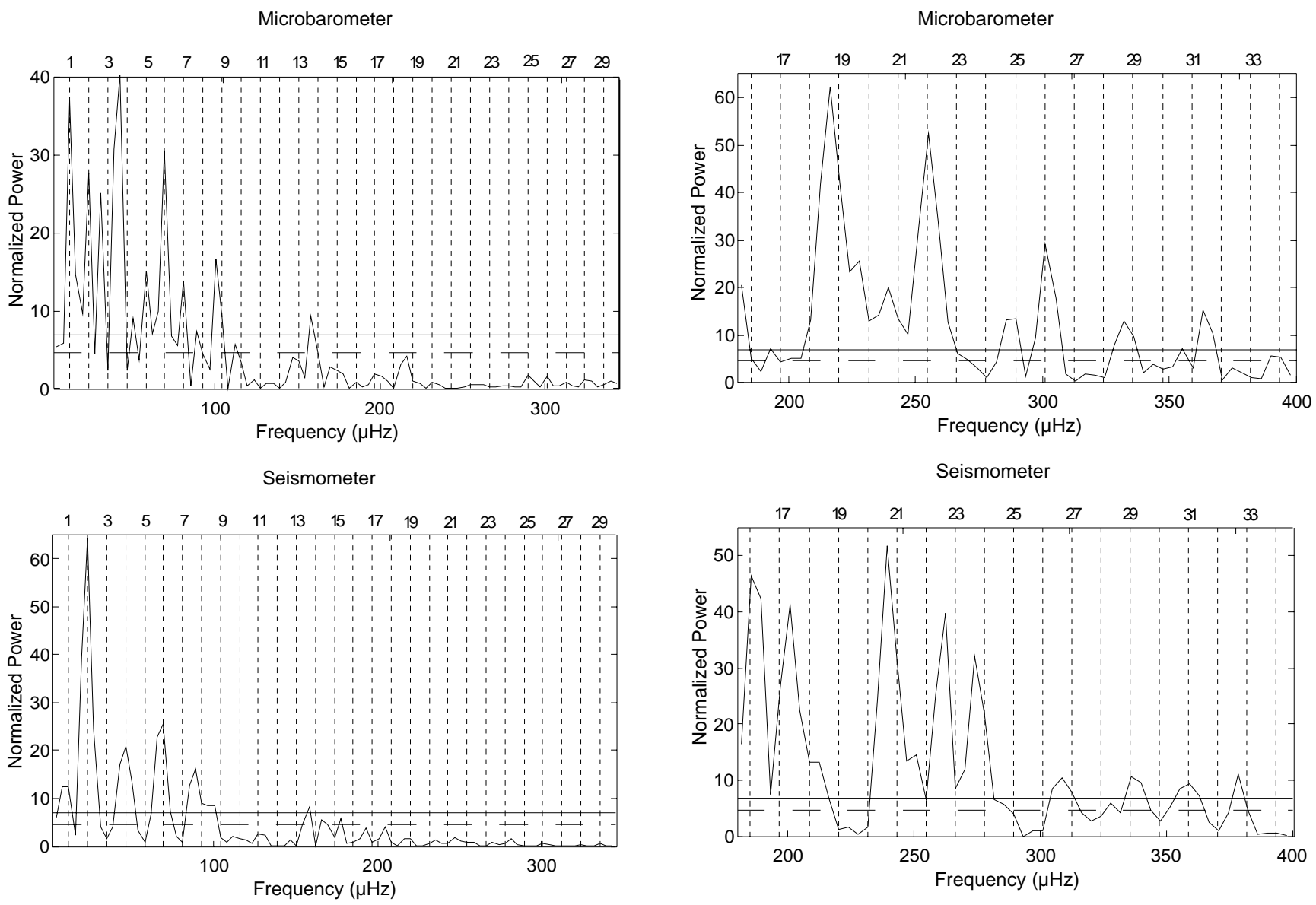

Fig. 5. Power frequency spectra based on the raw records for 36$72 \mathrm{~h}$ time segment from Fig. 4. The horizontal lines show the $99 \%$ (dashed) and $99.9 \%$ (solid) confidence levels. The vertical dashed lines correspond to the frequencies of solar tide subharmonics. The numbers at these lines indicate subharmonic numbers.

4. On the average, the oscillation amplitude decreases sharply for frequencies above $\sim 300 \mu \mathrm{Hz}$.

\subsection{Correlation coefficients}

We have calculated the following correlation coefficients:

$\overline{N_{h, p}^{\prime} N_{l, p}^{\prime}} / \sqrt{\overline{N_{h, p}^{\prime 2}} \overline{N_{l, p}^{\prime 2}}}=0.46 \quad \overline{N_{h, s}^{\prime} N_{l, s}^{\prime}} / \sqrt{\overline{N_{h, s}^{\prime 2}} \overline{N_{l, s}^{\prime 2}}}=0.36$

$\overline{N_{h, p}^{\prime} N_{h, s}^{\prime}} / \sqrt{\overline{N_{h, p}^{\prime 2}} \overline{N_{h, s}^{\prime 2}}}=0.13 \quad \overline{N_{l, p}^{\prime} N_{l, s}^{\prime}} / \sqrt{\overline{N_{l, p}^{\prime 2}} \overline{N_{l, s}^{\prime 2}}}=0.19$,

where the indexes $h$ and $l$ correspond to high-and lowfrequency subranges, respectively, the indexes $p$ and $s$ correspond to microbarometer and seismometer spectra, respectively, $N^{\prime}=N-\bar{N}$, and the bar denotes the average over time of the observation. The coefficient magnitudes are based on about 500 values of $N$ for each instrument in each frequency subrange.

Fig. 6. Power frequency spectra based on the filtered series for 60 $96 \mathrm{~h}$ time segment from Fig. 3. The remaining designations are the same in Fig. 5.

The correlation coefficients presenting a relationship between the $N$ values of simultaneous spectra for the highand low-frequency subranges are substantial. The correlation coefficients between the $N$ values of simultaneous microbarometer and seismometer spectra are low. Thus, although both the microbarometer and seismometer spectra show the above-mentioned common features, the spectra for simultaneous measurements are usually significantly distinct from each other. This is hardly surprising since the subject of our interest is very weak signals detected by the measurements of different physical quantities on the background of different interferences.

\subsection{Effect of macro-scale processes}

As indicated in the Introduction, the axial components of atmospheric angular momentum $M$, associated with zonal wind, for the Northern and Southern Hemispheres $\left(M_{\mathrm{NH}}\right.$ and $M_{\mathrm{SH}}$, respectively) are used to present atmospheric state on a global scale. We employ the $M_{\mathrm{NH}}$ and $M_{\mathrm{SH}}$ magnitudes calculated by Sidorenkov (2002) at 00:00, 06:00, 12:00, and 


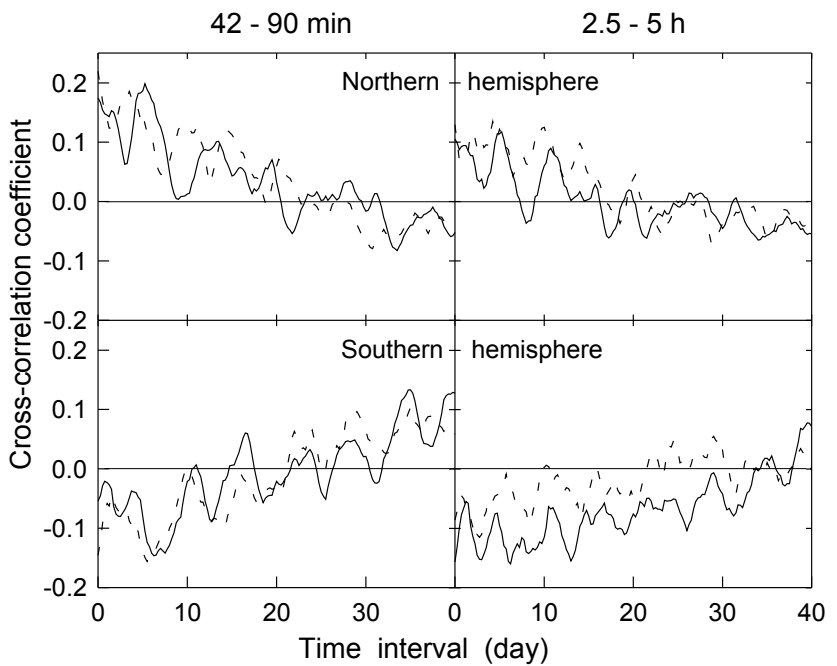

Fig. 7. Cross-correlation coefficients between the components of atmospheric axial angular momentum, associated with zonal wind, for the Northern and Southern Hemispheres and the numbers of prominent peaks in power spectra derived from microbarometer (solid lines) and seismometer (dashed lines) measurements for the period ranges of $42-90 \mathrm{~min}$ and $2.5-5 \mathrm{~h}$.

18:00 UTC on the base of the NCEP/NCAR reanalysis wind data. To find a relation between the considered oscillation (wave) perturbation and macro-scale dynamic processes, a temporal dependence of the cross-correlation coefficients,

$R(\Delta t)=\overline{N^{\prime}(t) M^{\prime}(t+\Delta t)} / \sqrt{\overline{N^{\prime 2}} \overline{M^{\prime 2}}}$,

between the $M_{\mathrm{NH}}$ and $M_{\mathrm{SH}}$ values and the $N$ numbers of both the subranges for both the instruments are calculated (Fig. 7). Here, $M^{\prime}=M-\bar{M}, t$ is the time, and $\Delta t$ is the time interval between observed $N$ and $M$ values. The absolute magnitudes of $R$ are small $(|R| \leq 0.2)$. This means the excitation of most oscillations is not associated with atmospheric state on a global scale. The coherence spectra derived from the $R(\Delta t)$ functions are given in Fig. 8. The seven spectra out of eight show a prominent peak about the 5 -day period. The exception is the spectrum for correlation between $M_{\mathrm{NH}}$ and $N$ numbers for the high-frequency subrange and seismometer measurements.

\subsection{Effect of tropical cyclones}

Global data on tropical cyclones (TC) with wind speeds above $30 \mathrm{~m} \mathrm{~s}^{-1}$ are taken from Pokrovskaya and Sharkov (2001). Such TCs were observed for the most part of the measurement time. Sometimes, two or even three TCs existed simultaneously around the globe. In the absence of TCs, the mean magnitudes of $N$ in the high-frequency subrange are 10.5 and 11.7 for the microbarometer and seismometer measurements, respectively, as compared with $N=$ 9.7 for both the instruments if there are TCs. Those for the

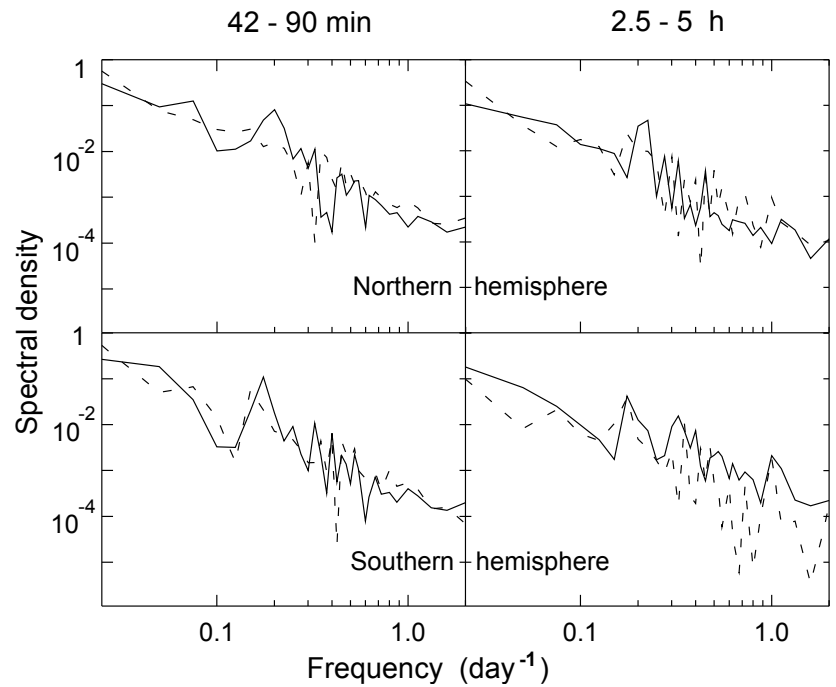

Fig. 8. Coherence spectra derived from the temporal dependence of cross-correlation coefficients, shown in Fig. 7. Solid and dashed lines denote the same as in Fig. 7.

low-frequency subrange are 3.9 and 4.2 as compared with 3.7 and 4.0 if there are TCs. So, the oscillation (wave) perturbation is found to be slightly weaker, when TCs exist in all the four cases.

\subsection{Effect of large earthquakes}

Data about large earthquakes with a magnitude $M \geq 6.5$ are available on the Internet at http://earthquake.usgs.gov/ earthquakes/eqarchives/significant/. There were 14 large earthquakes on the globe for the measurement time. We used the superposed epoch method in search of an earthquake influence on oscillation occurrence. For each earthquake, diurnal mean numbers $N$ were calculated for the first, second, and third days both before and after the earthquake occurrence. These $N$ values averaged over the 14 earthquakes are shown in Fig. 9. A weak minimum of $N$ is revealed before the earthquake occurrence in all the four cases. This means that the oscillation (wave) perturbation increases when earthquakes occur.

\section{Phase analysis of oscillations}

\subsection{Starting points}

We assume that the atmospheric waves studied can be quasisteady for some time. Then, the oscillations revealed may be approximated by sinusoids. An oscillation of surfacepressure, detected by microbarometer, is represented by

$p^{\prime}(t)=\delta p \sin \left(\omega t+\varphi_{p}\right)$,

where $\omega, \delta p$, and $\varphi_{p}$ are the angular frequency, amplitude, and phase of the oscillation, respectively. The seismometer 


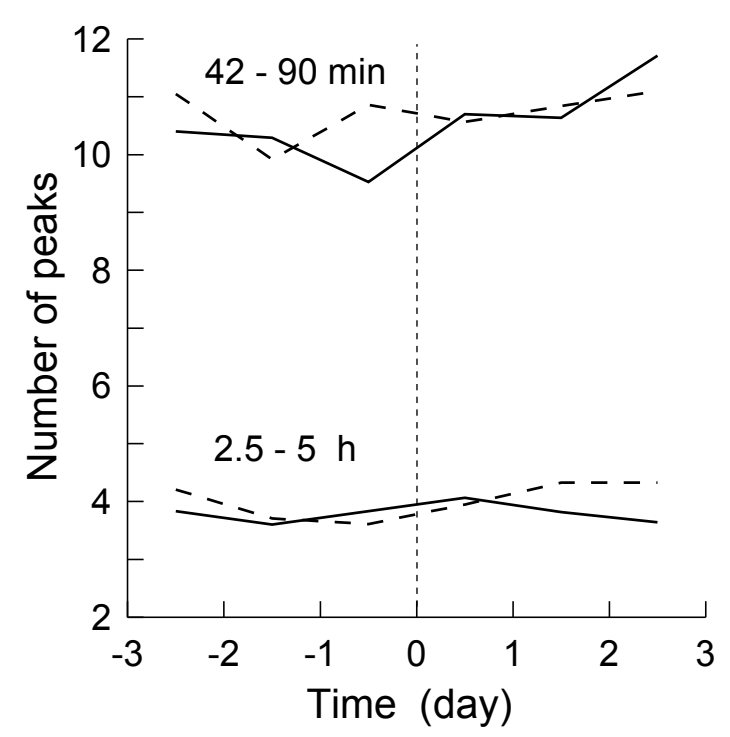

Fig. 9. Change in diurnal mean numbers of prominent spectral peaks around the occurrence time of large earthquake. The solid lines are for the numbers derived from the power spectra of groundpressure variations. The dashed lines are the same, but for the seismometer records. The zero time corresponds to earthquake occurrence time.

records the vertical displacement of sensor "mass" (pendulum), $\zeta$, relative to the ground. This displacement can be caused by both the vertical displacement $z^{\prime}$ of the earth's surface and pressure perturbation $p^{\prime}$ (see Appendix A). The $\zeta$ and $z^{\prime}$ displacements are also represented by sinusoids:

$\zeta(t)=\delta \zeta \sin \left(\omega t+\varphi_{\zeta}\right)$

and

$z^{\prime}(t)=\delta z \sin \left(\omega t+\varphi_{z}\right)$,

where $\delta \zeta$ and $\delta z$ are the amplitudes, and $\varphi_{\zeta}$ and $\varphi_{z}$ are the phases of appropriate displacements. The phase shifts

$\Delta \varphi=\varphi_{\zeta}-\varphi_{p}$

for oscillations detected simultaneously by microbarometer and seismometer are the subject of our interest.

\subsection{Procedure of phase analysis}

The autumn records of 1997 have been used to estimate the phase shifts $\Delta \varphi$. The couples of the weekly raw records (Sect. 2) were taken to calculate the cross-correlation functions between the microbarometer and seismometer signals. The coherence spectra in the $\sim 0.5-12 \mathrm{~h}$ period range were obtained from these functions using the Parzen window. The method of window closing was applied to find an optimal window width as a compromise between statistical significance of the revealed spectral peaks and frequency resolution. The reliability of the spectral peaks was determined by comparison the spectra with the coherence spectrum for the couple of statistically independent Gaussian processes taking into account a coherence coefficient expected due to the use of the Parzen window. The oscillations taken for the phase analysis satisfy the $90 \%$ confidence level of not being noise. Moreover, these have unchangeable locations in frequency at varying the window width over a wide range.

To decrease the influence of other peaks on the phase of a selected peak the raw records were subjected to a narrowband filtering which does not shift the oscillation phase. We used a Potter filter with a band-width which is connected with the width of a considered peak. So, for each selected peak, a special couple of microbarometer and seismometer series was prepared. To minimize the influence of the unsteadiness which can be of a common origin for the oscillations of pressure and gravity, the method of simultaneous processing the couples of series was used (Petrova et al., 1996). Also for this purpose, the estimations of phase shift $\Delta \varphi$ were made with a sliding temporal window of width being equal to the above-mentioned optimal width of the Parzen window.

The phase of signal at the output of both instruments is shifted due to a mechanical-electrical transductor and electronic amplifier. The $\omega$-dependences of this phase shift are different for the used microbarometer and seismometer. We attempted to correct the magnitudes of $\Delta \varphi$ for the instrumental phase shift. This is discussed in Sect. 5.

\subsection{Results}

The coherence spectra for 9 couples of simultaneous weekly records have resulted in 90 statistically reliable oscillations in the $\sim 0.5-12 \mathrm{~h}$ period range. The frequencies for half of these oscillations are displaced from the frequencies of the $\mathrm{S}_{m}$ subharmonics with $m$ from 2 to $\sim 45$ no more than by $2 \mu \mathrm{Hz}$. Note that there are oscillation couples with frequencies differing by a factor of 2 in the majority of the spectra. Such couples are about one third of the revealed oscillations.

For the majority of the oscillations, sufficiently long periods are revealed when $\Delta \varphi$ changes little with $t$ (less than $\pi / 4$ from a mean magnitude). Examples of such $\Delta \varphi(t)$ are shown in Fig. 10. The oscillations taken for the analysis have either a quasi-steady behavior of $\Delta \varphi$ with time for no less than 2 days, or a strong perturbation of $\Delta \varphi$ for no more than 1 day. The phase shifts $\Delta \varphi$ for 80 oscillations satisfy of these conditions. The values of $\Delta \varphi$, averaged over time interval(s) of quasi-steady behavior of $\Delta \varphi$, are plotted in Fig. 11. The points in the figure are clustered. That indicates some $\omega$ dependence of $\Delta \varphi$, discussed in Sect. 5. Only four points (at $152,182,244$, and $287 \mu \mathrm{Hz}$ ) certainly lie outside the cluster up to an angle of $\sim \pi / 2$ for the former two points. 
(a)

$\mu \mathrm{Hz} \quad \mathrm{m}$

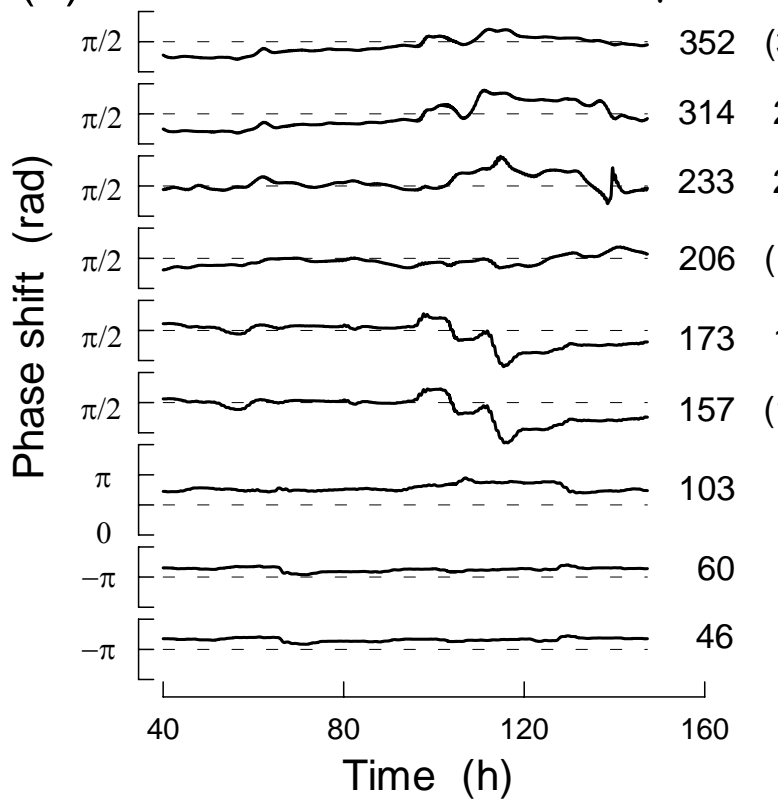

(b)

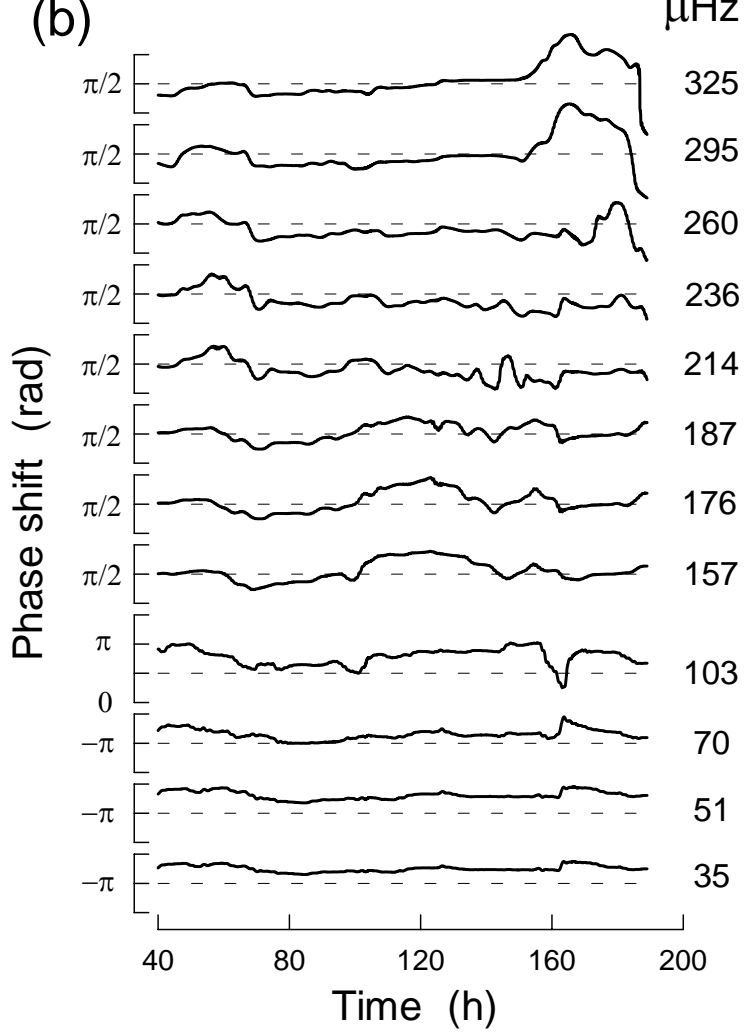

Fig. 10. Phase shift between oscillations, detected simultaneously by microbarometer and seismometer measurements during 17-23 October (a) and 28 October-4 November (b) 1997. The time is counted from the beginning of records. The numbers at a line indicate both the frequency of oscillations and the number of solar tidal subharmonic which is the closest to the oscillation in frequency. This number is bracketed if the oscillation frequency is displaced from the subharmonic frequency more than $2 \mu \mathrm{Hz}$.

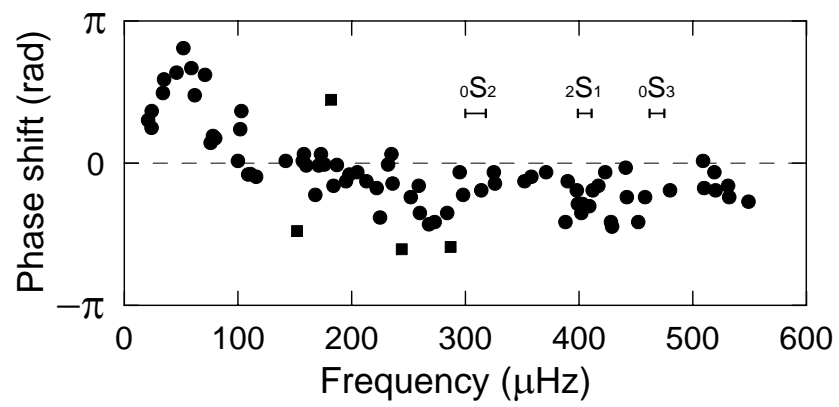

Fig. 11. Phase shift between oscillations, detected simultaneously by microbarometer and seismometer measurements, as a function of frequency (circles). The phase shifts which certainly are not in agreement with characteristic ones are represented by squares. The frequency ranges of the Earth free spheroidal oscillations which are split into components (Rosat et al., 2005) are marked by bars.

\section{Discussion}

Although the Earth's free oscillations, the gravity tides due to the Moon and the Sun, and the hydrology and ocean loading contribute to the perturbations of surface-gravity at periods longer than $0.5 \mathrm{~h}$, the dominant contribution is from atmospheric motions. This is clearly demonstrated by the behavior of signal in time for simultaneous seismometer and barometer records (see, e.g., raw records shown by Müller and Zürn, 1983, and Garmash et al., 1989). Besides the $\mathrm{S}_{m}$ subharmonics and the normal modes, intensive IGWs from local atmospheric sources contribute to the perturbations of both pressure and gravity in the $\sim 0.5-5 \mathrm{~h}$ period range. The primary causes of IGWs are believed to be topography, frontal and convective activity, geostrophic adjustment, mesospheric vortices, and wind shear (e.g., Gossard and Hooke, 1975; Fritts and Alexander, 2003). The IGW frequencies are not fixed: they depend on the parameters of wave sources undergoing random variations and/or depending on the variable state of the atmosphere. As a rule, a local atmospheric perturbation of pressure, which emits a wave, disappears due to the emission of this wave, and wind changes continuously with synoptic setting in the case of mountain waves. This is why IGW at a specified frequency is usually observed over a time interval of no more than several wave periods. Hence, IGW spectral peaks are expected to be weak in comparison with peaks from steady-frequency global waves if the length of analyzed record is much longer than IGW lifetimes. Thus, IGWs are not detected in spectra for records with the length of several days and therefore not revealed in the coherence spectra obtained in Sect. 4. However, IGW signs can be included in the dynamic spectra of Sect. 3, derived from shorter records.

Therefore, global atmospheric waves at fixed frequencies with lifetimes of no less than several days may be assumed to be revealed in the coherence spectra presented in Sect. 4 . 
We will discuss these waves in some details below. Here it may be sufficient to note that the high $\mathrm{S}_{m}$ subharmonics are certainly revealed (see Introduction). First of all, we will discuss our results on the phase shift $\Delta \varphi$.

Basing on simultaneous barometric and gravimetric measurements, Warburton and Goodkind (1977) and Crossley et al. (1995) have shown that the $S_{m}$ oscillations of gravity force $\left(f_{\mathrm{g}}^{\prime}\right)$ and surface-pressure $\left(p^{\prime}\right)$ are almost in phase for $m \geq 3$ if $f_{\mathrm{g}}^{\prime}$ is assumed positive in the "upward" direction. Spratt (1982) has explained this result, decomposing the perturbations of different physical values on the earth's surface into spherical harmonics. He used the spherical, horizontally uniform, oceanless model of the Earth and assumed the static deformation of elastic sphere by loading it from the $p^{\prime}$-field of the atmospheric tide. The assumption of static condition is justified by the fast response of the Earth's crust to the $p^{\prime}$-perturbations since the seismic wave velocity, as a rule, exceeds many-fold the sound speed in the atmosphere. Spratt (1982) has derived the transfer coefficient between the perturbations of $p^{\prime}$ and $f_{\mathrm{g}}^{\prime}$,

$$
L=f_{\mathrm{g}}^{\prime} / p^{\prime},
$$

for a spherical harmonic of degree $n$ :

$$
L_{n}=\frac{2 \pi G}{g}\left(\frac{2}{2 n+1}\right)\left[n-(n+1) k_{n}+2 h_{n}\right],
$$

where $G$ is the gravitational constant, $g$ is the gravity acceleration, and $k_{n}$ and $h_{n}$ are the surface loading Love numbers $\left(k_{n}>0\right.$, but $\left.h_{n}<0\right)$. The first term in Eq. (6) corresponds to the direct Newtonian attraction by the atmospheric masses, and the other two correspond to the effects of the deformation (see Appendix A for details). The first term turns out to be dominant for any $n$ from $n=1$, the predominance increasing with $n$. The $L_{n}$ magnitude approaches $2 \pi G / g=4.27 \times 10^{-7} \mathrm{Gal} \mathrm{mb}^{-1}$ as $n \rightarrow \infty$. Therefore, the magnitudes of $L$ are always positive and the oscillations of $f_{\mathrm{g}}^{\prime}$ and $p^{\prime}$, caused by $\mathrm{S}_{m}$ subharmonics, should correspondingly be in phase. The use of different models for the ocean response to atmospheric forcing does not change the inference (Boy et al., 1998).

The approach to $f_{\mathrm{g}}^{\prime}$ estimation, adopted by Spratt (1982) for the $S_{m}$ subharmonics, is suitable for any atmospheric global wave in the considered period $(\tau)$ range (up to $\tau \sim$ $0.5 \mathrm{~h}$ ). We use this approach to estimate phase shifts $\Delta \varphi$ between oscillations detected simultaneously by microbarometer and seismometer (Sect. 4). The three terms of Eq. (A8), which describe the $f_{\mathrm{g}}^{\prime}$-perturbation, may be presented as

$C_{g a} p^{\prime}+\left(C_{k}+C_{h}\right) z^{\prime}=L p^{\prime}$.

Then, the Eq. (A8) of motion for the sensor "mass" of seismometer becomes

$\omega_{0 g}^{2} \zeta=\omega^{2} z^{\prime}+\left(L+C_{b}\right) p^{\prime}$.
For the considered static deformation, a $p^{\prime}$-oscillation and the $z^{\prime}$-oscillation induced by it are opposite in phase $\left(z^{\prime} \propto\right.$ $\left.-p^{\prime}\right)$. Since $\left(L+C_{b}\right)>0$, as is seen from Eq. (8), $\zeta$ and $p^{\prime}$ can be both in phase and in antiphase. Therefore, we may expect the $\Delta \varphi$ magnitudes of both around 0 and $\pm \pi$. However, $\Delta \varphi$ as a function of $\omega$ (Fig. 9) does not show any abrupt jumps of $\sim \pi$ : the function is well monotone. Next we present arguments which forced us to chose zero as expected $\Delta \varphi$ for the detected oscillations interpreted as atmospheric global waves.

First we consider the case of perfect shielding of the seismometer from a direct effect of atmospheric processes $\left(C_{b}=\right.$ 0 ). To make a comparison between $\omega^{2} z^{\prime}$ and $L p^{\prime}$ in Eq. (8), the transfer coefficient between the perturbations of $p^{\prime}$ and $z^{\prime}$

$\vartheta=z^{\prime} / p^{\prime}$,

is used. This coefficient for a spherical harmonic of degree $n$ is

$\vartheta_{n}=\frac{4 \pi G r_{0} h_{n}}{(2 n+1) g^{2}}$,

where $r_{0}$ is the Earth's radius (Spratt, 1982). Using the $h_{n}$ magnitudes from Farrell (1972), we obtain the decrease of $\vartheta_{n}$ with $n$ from a maximum at $n=2$ to 0 as $n \rightarrow \infty$. The comparison of $\omega^{2} \vartheta_{n}$ with $L_{n}$ results in the inequality $\left|\omega^{2} \vartheta_{n}\right|<L_{n}$ at $n \geq 17$ for any magnitude of $\omega$ in the considered period range. The atmospheric normal Lamb modes represented by the spherical harmonics of such degrees $n$ are of frequencies exceeding $\sim 150 \mu \mathrm{Hz}(\tau \leq 2 \mathrm{~h}$ ) (Dikii, 1965; see also Garmash et al., 1989). However, dominant spherical harmonics in the expansions of all the $S_{m}$ subharmonics are unknown. In this case, the assumption $\Delta \varphi=0$ is based on the above-mentioned observed closeness of $f_{\mathrm{g}}^{\prime}$ - and $p^{\prime}$ oscillation phases for $m$ from 3 to $\sim 10$, which should be interpreted as $\left|\omega^{2} z^{\prime}\right|<\left|L p^{\prime}\right|$.

It should be also noted that the chamber enclosing the used seismometer is not perfectly hermetic (Lin'kov, 1987). The direct action of atmospheric waves on the seismometer through variable buoyancy (see Appendix A for details) obviously increases with $\tau$. The validity of protection from direct action of $p^{\prime}$-perturbations has been tested only for $\tau \leq 2 \mathrm{~h}$. Since $C_{b}>0$, it should not be ruled out that the buoyancy effect can result in the $\zeta$-oscillation phases as for the $p^{\prime}$ oscillations for $\tau>2 \mathrm{~h}$.

Our attempt to eliminate an instrumental phase shift was successful for the frequency range of $\sim 100-200 \mu \mathrm{Hz}$ ( $\tau \sim$ $1.5-2.5 \mathrm{~h}$ ), where $\Delta \varphi$ is close to zero within the accuracy of the analysis. We were, however, not successful in the low-frequency range $(\tau>2.5 \mathrm{~h})$, where the $\Delta \varphi$ magnitudes near zero are mainly expected. In the high-frequency range $(\tau<1.5 \mathrm{~h})$, the $\Delta \varphi$ magnitudes are negative and clustered around $-\pi / 4$. The instrumental phase shift is believed to be not fully eliminated in this frequency range. We suppose that the phase shifts $\Delta \varphi$ for global atmospheric waves are close to 
zero in the high-frequency range too. However, an alternative explanation for origin of oscillations in the high-frequency region should also be considered.

This alternative explanation bases on an assumption that there are seismic oscillations (waves) at steady frequencies, which penetrate into the atmosphere by the piston mechanism. In this case, a $z^{\prime}$-oscillation generated in the solid Earth induces the $p^{\prime}$-oscillation, and the vertical flux of wave energy, $F$, is upward-directed from the surface to the atmosphere $(F>0)$. The flux $F$ is determined by

$F=\overline{p^{\prime} w^{\prime}}$,

where $p^{\prime}$ and $w^{\prime}$ are the wave perturbations of pressure and vertical velocity in fluid, respectively, and the bar denotes the average over time. At the surface, $p^{\prime}$ is the measured quantity, and $w^{\prime}$ is the measured vertical velocity of surface displacement $\left(w^{\prime}=d z^{\prime} / d t\right)$. Equations (1), (3), and (11) give

$F=\frac{\omega \delta p \delta z}{2} \sin \left(\varphi_{p}-\varphi_{z}\right)$

The condition $F>0$ is fulfilled if the phase shift $\left(\varphi_{p}-\varphi_{z}\right)$ is in the range between 0 and $\pi$.

Garmash et al. (1989) have derived the transfer coefficients between the oscillations of $z^{\prime}$ and $p^{\prime}$,

$\eta=p^{\prime} / z^{\prime}$,

if a global seismic wave induces a global atmospheric wave, and the atmosphere is in the hydrostatic equilibrium. In the absence of resonance, they have obtained

$\eta=p_{0} / H \approx 13 \mathrm{~Pa} \mathrm{~m}^{-1}$,

where $p_{0}$ is the standard atmospheric pressure and $H$ is the pressure scale height. In accordance with Eq. (6), the contribution of the Newtonian attraction by the atmospheric masses to the $L$ magnitude does not exceed $2 \pi G / g$. As the inequality $(2 \pi G / g) \cdot\left(p_{0} / H\right) \ll \omega^{2}$ is fulfilled in the considered period range, the Newtonian attraction may be neglected in Eq. (8) for waves of seismic origin. However, the deformation perturbation of gravity force, $f_{g d}^{\prime}$, (see Appendix A for details) remains. As the seismometer used is well protected from a direct effect of variations in the ambient air for $\tau \leq 2 \mathrm{~h}$, Eq. (8) is simplified to

$\omega_{0}^{2} \zeta=\left(C_{k}+C_{h}+\omega^{2}\right) z^{\prime}$

for waves of seismic origin. The oscillations $f_{g d}^{\prime}$ and $z^{\prime}$ is in phase because of the decrease (increase) of $g$ at $z^{\prime}>0$ $\left(z^{\prime}<0\right)$. This is why the sum $C_{k}+C_{h}$ is positive and $\varphi_{\zeta}$ is equal to $\varphi_{z}$. Hence, the phase shift $\Delta \varphi$ determined by Eq. (4) lies between $-\pi$ and 0 if an oscillation is generated in the solid Earth. Of the oscillations with $\Delta \varphi<0$, three of the ones denoted by squares in Fig. 11 are most likely seismic waves penetrating the atmosphere. (Note, however, that an explanation for the oscillation at $182 \mu \mathrm{Hz}$ with $\Delta \varphi \sim \pi / 2$, which is also denoted by square, has not been found.) In the following, we consider possible origins of seismic waves at steady frequencies in the $\sim 0.5-2 \mathrm{~h}$ period range $(\sim 150-$ $550 \mu \mathrm{Hz}$ ).

The Earth's free spheroidal oscillations of ${ }_{0} \mathrm{~S}_{2},{ }_{2} \mathrm{~S}_{1}$, and ${ }_{0} \mathrm{~S}_{3}$ fall in the high-frequency part of this period region (e.g., Rosat et al., 2005). The Earth's free oscillations are shown to occur not only after large earthquakes, but also on seismically quiet days (e.g., Lin'kov et al., 1991; Davydov and Dolgikh, 1997; Nawa et al., 1998). Measurements of surfacepressure variation have revealed the penetration the ${ }_{0} \mathrm{~S}_{2}$ oscillation at a period of $54 \mathrm{~min}(309 \mu \mathrm{Hz})$ to the atmosphere even on seismically quiet days (Garmash et al., 1989; Lin'kov et al., 1991; Shved et al., 2000). As is seen from Fig. 11, the ${ }_{0} S_{2}$ and ${ }_{0} S_{3}$ oscillations likely turn out to be too weak to be derived from the coherence spectra obtained here. However, the cluster of oscillations in the frequency region of the ${ }_{2} \mathrm{~S}_{1}$ multiplet at the period of $41 \mathrm{~min}(405 \mu \mathrm{Hz})$ is possibly a signature of the ${ }_{2} \mathrm{~S}_{1}$ oscillation. It should be noted that the penetration of the spheroidal free oscillations into the atmosphere is owing not only by the piston mechanism, but also due to a change in the earth's gravitational field of the deformed earth (e.g., Lognonné et al., 1998; Shved et al., 2000). In this case, the phase shift $\Delta \varphi$ can be different from the one obtained here under the piston mechanism assumption.

Water accumulation in a part of sea or lake forces standing waves (seiches) which are the free oscillations of entirely or partly closed water-basins (e.g., Defant, 1960). Seismic oscillations caused by seiches are detected at sites close to sea (e.g., Davydov and Dolgikh, 1995). Our measurements are performed at the distance of about $2.5 \mathrm{~km}$ from the Gulf of Finland and about $75 \mathrm{~km}$ from lake Ladoga. Malinina (1966) observed the Ladoga seiches to have $340,280,195,131,88$, $56,28,16$, and 10 min periods with durations of $2-\sim 30$ wave periods and amplitudes of the water-level oscillation up to $15 \mathrm{~cm}$. But a seiche spectrum for the Gulf of Finland was not likely measured. Thus, it cannot be ruled out that certain steady-frequency seismic oscillations penetrating the atmosphere are caused by seiches. A more detailed examination is needed to reveal seiches among the detected oscillations.

Petrova (2002) has proposed to associate certain seismic oscillations in the $\sim 1-5 \mathrm{~h}$ period range with free flexural oscillations of the lithospheric plates. At present there is no evidence in favour of this hypothesis. However, to discard such possible source of common oscillations of the earth's surface and atmosphere would be unreasonable.

We are now discussing global atmospheric waves at steady frequency, obtained from our microbarometer and seismometer records. As pointed out above, these waves are represented by the $S_{m}$ subharmonics and the normal modes. There are two competing mechanisms of generation for the considered short-period tidal subharmonics: direct thermal excitation or nonlinear interactions between a long-period one and another global wave. The normal modes are only excited due to nonlinear dynamic processes. Examples of 
nonlinear processes which generate tidal and normal modes are interactions of a low subharmonic of the migrating tide with a stationary planetary wave (e.g., Angelats i Coll and Forbes, 2002), normal mode (e.g., Pancheva et al., 2002; Wu et al., 2002), or another tidal subharmonic (e.g., Akmaev, 2001). So, the excitation of short-period tidal and normal modes depends on the thermal or dynamic state of the atmosphere, which both are highly variable. These modes should therefore be transient. Both the dynamic spectra (Sect. 3) and the behavior of the phase shift in time (Sect. 4) show that the waves considered can exist for several days. The strong variations in oscillation frequency, shown by the dynamic spectra (see Figs. 3 and 4), can be explained as a consequence of transience of wave source, which consists in a relative change in intensity of waves during their generation. This means that the frequency variations are rather spurious. The substantial correlation between the wave perturbations of the high- and low-frequency subranges (Sect. 3) can be explained by the capacity of nonlinear processes to generate a set of waves in a wide period range simultaneously.

The present study advances in the knowledge of the high $\mathrm{S}_{m}$ subharmonics: both the appearance of sequences of the $\mathrm{S}_{m}$ subharmonics in the dynamic spectra (Sect. 3) and the detection of ones in the coherence spectra (Sect. 4) evidence their excitation up to $m \sim 30$.

As there are no realistic theoretical estimates of normal mode frequencies for the considered period range, it would be risky to correlate any oscillation detected by us with a normal mode. However, one may think that the normal modes are present in our spectra. This assumption is supported not only by the $8-\mu \mathrm{Hz}$ periodicity signs in the spectra derived from microbarometer and seismometer measurements (see Introduction), but also by the following findings. Petrova and Lyubimtsev (2006) have detected oscillations with the same steady frequencies in the $\sim 30-100 \mu \mathrm{Hz}$ range from measurements by vertical-pendulum seismometers at ten sites spatially located from $46.4^{\circ} \mathrm{S}$ to $59.9^{\circ} \mathrm{N}$ in latitude and from $17.0^{\circ} \mathrm{W}$ to $137.0^{\circ} \mathrm{E}$ in longitude. They have obtained the frequency spectra for records of about 8 days of length and a combined duration from 21 to 60 days, depending on site. Besides all the $S_{m}$ subharmonics $(m=4-8)$, the steadyfrequency oscillations at about $49,54,64,74,77,86$, and $91 \mu \mathrm{Hz}$ have simultaneously been detected at two or more sites. The steady-frequency oscillations at 64,74 , and $77 \mu \mathrm{Hz}$ have also been revealed by Karpova et al. (2004) through the technique of spectra multiplication. Moreover, the 74 and $77 \mu \mathrm{Hz}$ oscillations have been earlier detected from European superconducting gravimeter measurements (Smylie et al., 1993). It appears reasonable to assume that there are atmospheric normal modes among the above-mentioned oscillations.

Gerrard et al. (2010) have obtained time series of photometer measurements of the thermospheric high-latitude emission at South Pole Station $\left(74^{\circ} \mathrm{S}, 19^{\circ} \mathrm{E}\right)$ and McMurdo Station $\left(78^{\circ} \mathrm{S}, 167^{\circ} \mathrm{E}\right)$ for three winter periods. The power spectra have been computed in the $2-12 \mathrm{~h}$ period range using the sliding data window of 7 days in length with 1-day step. The dynamic spectra obtained look like our dynamic spectra (Sect. 3) in the following principal features. (i) The oscillations are detected as sets of spectral peaks separated by nearly equal spacing in frequency. (ii) These oscillations last usually many days (a month and up). (iii) There are oscillations with a strong variation of frequency with time. Since the amplitude of global wave increases with height in dissipationless atmosphere (e.g., Forbes, 1995) one would expect that the oscillations detected by Gerrard et al. (2010) are $\mathbf{S}_{m}$ subharmonics and normal modes.

There is a prominent 5-day peak in the coherence spectra derived in Sect. 3 (see Fig. 8). Earlier, Boy et al. (1998) have also obtained a maximum around the 5-day period for spectral coherence between surface-pressure and gravity, using 10-year measurements at Strasbourg. Dickey et al. (1992) have revealed the same feature in spectral coherence between length-of-day and axial angular momentum of the entire atmosphere. This feature may be considered as evidence that there are global dynamic processes in the atmosphere on the time scale of $\sim 5$ days. The 5-day normal Rossby modes observed (e.g., Forbes, 1995; Madden, 2007) may be reasonably assumed to be such a processes. The manifestation of a 5-day feature in our coherence spectra might be accounted for in two ways. First, perhaps, there are time spans when the atmospheric state turns out to be favorable for a simultaneous generation of both the 5-day wave and atmospheric normal modes in the $\sim 1-5 \mathrm{~h}$ period range. Second, the occurrence of a 5-day wave may change the conditions required for generation of $S_{m}$ subharmonics and (or) normal modes at these periods.

We have derived a slight reduction in the oscillation number $N$ during TC occurrence. A hypothesis explaining this result may be proposed if the detected oscillations are caused by global waves. One should take into account that each global wave is characterized by a strictly specified spatial configuration of the oscillating geopotential and wind fields. This is why transient processes in the background atmosphere can hinder to form this configuration. The closer the horizontal scale of a transient process to that of a global wave, the stronger is obviously its influence on the wave. The TCs are intense vortexes traveling on the globe. They therefore result in very strong transient perturbations of background atmosphere in temperature, pressure, and wind on the horizontal scale from $\sim 100 \mathrm{~km}$ up to $1000 \mathrm{~km}$ (e.g., Weatherford and Gray, 1988). We adopt the zonal length wave as a measure for the horizontal scale of global waves. If a detected high $\mathrm{S}_{m}$ subharmonic is the migrating tide, its zonal wave number $s$ is equal to its number $m$. The numbers $s$ from 5 to $\sim 30$ are therefore possible for $S_{m}$ at $\tau \sim 1-5 \mathrm{~h}$. As for the gravitational normal modes at identical periods, their horizontal configurations are described by the spherical surface functions of degree $n$ from 7 to $\sim 50$ (Karpova et al., 2002). This is also the possible range of $s$, since $s \leq n$. Thus, 
the horizontal scales of TCs are identical to those of many of the global waves. We therefore assume that the TCs can prevent a generation of the waves.

A weak increase of oscillation (wave) perturbation is detected around the occurrence time of large earthquakes in both the frequency subranges from both the microbarometer and seismometer measurements (see Fig. 9). As noted in the above discussion about the phase shift $\Delta \varphi$, the penetration of waves from the solid Earth into the atmosphere is possible. It is known that the Earth's free oscillations are considerably enhanced after large earthquakes (e.g., Rosat et al., 2005). However, we have not revealed any correlation between earthquake occurrence and detection of oscillations falling within and near the frequency ranges of the ${ }_{0} S_{2}$, ${ }_{2} \mathrm{~S}_{1}$, and ${ }_{0} \mathrm{~S}_{3}$ multiplets. One may assume that the increase of wave perturbation around earthquake occurrence time is caused by an excitation of the above-mentioned hypothetical flexural oscillations of the lithospheric plates. In any case, the origin of this increase remains unexplained. What is more, this increase needs in itself a further verification based on longer records.

\section{Conclusions}

This paper presents new evidences that measurements by seismological instruments can be very useful for investigation of atmospheric short-period waves with steady frequencies. Simultaneous co-located microbarometer and seismometer (gravimeter) measurements are particularly promising in this respect. To call attention of the researchers specializing in atmospheric dynamics to the use of these measurements we describe here the mechanisms of atmospheric perturbation action on sensor "mass" of seismometer in sufficient detail. As an example, the phase analysis of oscillations detected by both microbarometer and seismometer makes it possible to reveal the penetration of seismic waves into the atmosphere by the piston mechanism. The main results obtained in this paper are as follows.

1. Analysis of the microbarometer and vertical-pendulum seismometer records shows steady-frequency oscillations at intradiurnal periods up to the $\sim 0.5 \mathrm{~h}$ period, supporting the findings obtained earlier by Karpova et al. (2002, 2004). There are oscillations lasting for several days that agrees with the observations of waves in the thermosphere by Gerrard et al. (2010). As for the phase shift between oscillations detected simultaneously by the microbarometer and seismometer, in most cases there are time intervals from two to several days when that varies slightly.

2. Signatures of sequences of the solar tide subharmonics $\mathrm{S}_{m}$ are revealed up to $m \sim 30$. That means an increase of observed high $S_{m}$ subharmonics as compared with the measurements of Florsch et al. (1995) by a superconducting gravimeter, which are limited by the $S_{23}$ detection.

3. Some relationship of atmospheric global waves in the $\sim 1-5 \mathrm{~h}$ period range with the macroscale dynamic processes as the 5-day planetary waves and tropical cyclones is detected.

4. A weak increase of oscillation (wave) perturbation at the $\sim 1-5 \mathrm{~h}$ periods around the occurrence time of large earthquakes is revealed. However, at present we do not have an explanation of this phenomenon.

\section{Appendix A}

\section{Equation of motion for a linear vertical pendulum of seismometer}

The simplest physical model for an inertial seismometer is a sensor "mass"-and-spring system oscillating in a vertical direction, neglecting viscous damping. The equation of motion for the sensor "mass" is

$\frac{d^{2} \zeta}{d t^{2}}+\omega_{0}^{2} \zeta=f^{\prime}-\frac{d^{2} z^{\prime}}{d t^{2}}$

where $t$ is the time, $\zeta$ is the vertical displacement of the "mass" relative to the ground, $z^{\prime}$ is the vertical displacement of the earth's surface, $\omega_{0}$ is the angular eigenfrequency of the pendulum, and $f^{\prime}$ is the perturbation of external force acting on the "mass" (e.g., Wielandt, 2002). This perturbation is caused by both a change in gravity and a variable buoyancy of the "mass". Since vertical-pendulum seismometers detect oscillations of the earth's surface at periods longer than $1 \mathrm{~h}$ mainly through a gravity perturbation induced by these oscillations, the seismometers designed for revealing long-period oscillations are also named gravimeters.

The perturbation of the buoyancy force is

$f_{b}^{\prime}=g_{0} \rho^{\prime} / \rho_{M} \quad$,

where $\rho^{\prime}$ is the perturbation of air density, and $\rho_{M}$ is the "mass" density. A seismometer is generally placed indoors. The thermal regime for the seismometer may be therefore assumed to be independent on atmospheric processes. Thus, basing on the perfect gas law, the perturbation $\rho^{\prime}$ may be considered to be only dependent on the pressure perturbation $p^{\prime}$. Then, $f_{b}^{\prime}$ is represented as

$f_{b}^{\prime}=C_{b} p^{\prime}, \quad C_{b}=\chi \frac{g_{0} \rho_{0}}{p_{0} \rho_{M}}$,

$\rho_{0}$ and $p_{0}$ are the unperturbed density and pressure, respectively, and $\chi$ is the coefficient which characterizes the extent of seismometer protection from atmospheric pressure variations. The coefficient $\chi$ changes from zero in the case of 
perfect shielding to unity if there is no protection. This coefficient decreases obviously with the angular frequency $\omega$ of detected oscillation. The $\omega$-dependence of $\chi$ should be individually studied for each seismometer in its location.

The perturbation of gravity force, $f_{\mathrm{g}}^{\prime}$, which is caused by an atmospheric wave has contributions from both the direct Newtonian attraction of the "mass" by changing density of the atmosphere above the instrument (denoted as $f_{\mathrm{g} a}^{\prime}$ ) and the deformation of the Earth's crust through atmospheric loading (denoted as $f_{\mathrm{g} d}^{\prime}$ ) (e.g., Farrell, 1972; Spratt, 1982). The deformation contribution involves both a change in the earth's gravitational field of the deformed earth and a displacement of the "mass" through the gradient of this field (denoted as $f_{k}^{\prime}$ and $f_{h}^{\prime}$, respectively; $f_{\mathrm{g} d}^{\prime}=f_{k}^{\prime}+f_{h}^{\prime}$ ). In the hydrostatic approximation justified at wave periods of $\tau \geq 1 \mathrm{~h}$, the Newtonian attraction of the air is determined by the distribution of ground-pressure over the earth's surface. The wave perturbation $f_{\mathrm{g} a}^{\prime}$ may therefore be represented as

$f_{\mathrm{g} a}^{\prime}=C_{\mathrm{g} a} p^{\prime}$,

where $p^{\prime}$ is the wave surface-pressure perturbation at the location of seismometer and $C_{\mathrm{g} a}$ is the coefficient determined by $p^{\prime}$ distribution over the surface. The wave perturbations of $f_{k}^{\prime}$ and $f_{h}^{\prime}$ depend on the surface form resulting from variable atmospheric loading caused by a wave. These perturbations should therefore be proportional to the wave radial displacement of the surface, $z^{\prime}$, at the seismometer location:

$f_{k}^{\prime}=C_{k} z^{\prime}, \quad f_{h}^{\prime}=C_{h}\left(z^{\prime}+\zeta\right)$.

The coefficients of $C_{k}$ and $C_{h}$ are determined by the form of the deformed surface.

The $\zeta$ and $z^{\prime}$ oscillations with angular frequency $\omega$ are usually approximated by sinusoids. By using Eqs. (A2)-(A5), Eq. (A1) reduces then to

$$
\left(\omega_{0 g}^{2}-\omega^{2}\right) \zeta=\left(C_{k}+C_{h}+\omega^{2}\right) z^{\prime}+\left(C_{g a}+C_{b}\right) p^{\prime},
$$

where

$\omega_{0 g}^{2}=\omega_{0}^{2}-C_{h}$.

The estimation of both the dimensionless Love numbers (Farrell, 1972) and the ratio between amplitudes of wave perturbations in surface-pressure and vertical displacement of the surface (Sorrells, 1971) result in $C_{h} \ll \omega_{0}^{2}$. This gives $\omega_{0 g}^{2} \approx \omega_{0}^{2}$. For long-period oscillations with $\tau \geq 1 \mathrm{~h}$, the inequality of $\omega^{2} \ll \omega_{0 g}^{2}$ is fulfilled. (The inequality is fulfilled for the seismometer used in the present study since the pendulum eigenperiod is equal to $13 \mathrm{~s}$.) Equation (A6) is therefore simplified to

$\omega_{0 g}^{2} \zeta=\left(C_{k}+C_{h}+\omega^{2}\right) z^{\prime}+\left(C_{g a}+C_{b}\right) p^{\prime}$.

Acknowledgements. This work was supported by the Russian Foundation for Basic Research, projects 02-05-65299 and 07-0500475 , and by the "Development of scientific potential in higher school" program of Russian Ministry for Education and Science, project 2.1.1.4166. The authors are grateful to N. S. Sidorenkov for providing with the $M_{\mathrm{NH}}$ and $M_{\mathrm{SH}}$ data. We thank Ch. Jacobi and anonymous referees for their comments and suggestions that helped in improving the manuscript.

Topical Editor P. M. Ruti thanks two anonymous referees for their help in evaluating this paper.

\section{References}

Akmaev, R. A.: Seasonal variations of the terdiurnal tide in the mesosphere and lower thermosphere: a model study, Geophys. Res. Lett., 28, 3817-3820, 2001.

Angelats i Coll, M. and Forbes, J. M.: Nonlinear interactions in the upper atmosphere: The $s=1$ and $s=3$ nonmigrating semidiurnal tides, J. Geophys. Res., 107(A8), 1157, doi:10.1029/2001JA900179, 2002.

Boy, J.-P., Hinderer, J., and Gegout, P.: Global atmospheric loading and gravity, Phys. Earth Planet. Inter., 109, 161-177, 1998.

Crossley, D. J., Jensen, O. G., and Hinderer, J.: Effective barometric admittance and gravity residuals, Phys. Earth Planet. Inter., 90, 221-241, 1995.

Davydov, A. V. and Dolgikh, G. I.: Recording of long-period oscillations with a 52.5-m laser strainmeter, Fizika Zemli (Physics of the Earth), No. 3, 64-67, 1995.

Davydov, A. V. and Dolgikh, G. I.: Modulation of the free oscillations of the Earth, Izvestiya, Phys. Solid Earth, Engl. Transl., 33, 644-646, 1997.

Defant, A.: Physical oceanography, Vol. 2, Pergamon Press, New York, 1960.

Dickey, J. O., Marcus, S. L., Steppe, J. A., and Hide, R.: The Earth's angular momentum budget on subseasonal time scales, Science, 255, 321-324, 1992.

Dikii, L. A.: The terrestrial atmosphere as an oscillating system, Izvestiya, Atmos. Ocean. Phys., Engl. Transl., 1, 275-286, 1965.

Farrell, W. E.: Deformation of the Earth by surface loads, Rev. Geophys. Space Phys., 10, 761-797, 1972.

Florsch, N., Legros, H., and Hinderer, J.: The search for weak harmonic signals in a spectrum with application to gravity data, Phys. Earth Planet. Inter., 90, 197-210, 1995.

Forbes, J. M.: Tidal and planetary waves, in: Geophysical monograph, 87, edited by: Johnson, R. M. and Killeen, T. L., 67-87, 1995.

Forbes, J. M., Palo, S. E., and Marcos, F. A.: Longitudinal structures in lower thermosphere density, J. Geophys. Res. (A), 104, 4373-4385, 1999a.

Forbes, J. M., Palo, S. E., Zhang, X., Portnyagin, Yu. I., Makarov, N. A., and Merzlyakov, E. G.: Lamb waves in the lower thermosphere: Observational evidence and global consequences, J. Geophys. Res. (A), 104, 17107-17115, 1999 b.

Fritts, D. C. and Alexander, M. J.: Gravity wave dynamics and effects in the middle atmosphere, Rev. Geophys., 41(1), 1003, 2003.

Garmash, S. V., Lin'kov, E. M., Petrova, L. N., and Shved, G. M.: Generation of atmospheric oscillations by seismic-gravity oscillations of the Earth, Izvestiya, Atmos. Ocean. Phys., Engl. Transl., 25, 952-959, 1989.

Gerrard, A. J., Detrick, D., Mende, S. B., Lanzerotti, L. J., Weatherwax, A. T., and Bhattacharya, Y.: Photometric observations of 
630.0-nm OI and 427.8-nm $\mathrm{N}_{2}^{+}$emission from South Pole and McMurdo Stations during winter: Analysis of temporal variations spanning minutes to hourly timescales, J. Geophys. Res., 115, A08231, doi:10.1029/2009JA014970, 2010.

Gossard, E. E. and Hooke, W. N.: Waves in the atmosphere, Elsevier, Amsterdam, 1975.

Hamilton, K. and Garcia, R. R.: Theory and observations of the short-period normal mode oscillations of the atmosphere, J. Geophys. Res. (D), 91, 11867-11875, 1986.

Karpova, N. V., Petrova, L. N., and Shved, G. M.: Statistical study of seismic and ground pressure oscillations with steady frequencies in the $0.7-5 \mathrm{~h}$ period range, Ann. Geophys., 20, 823-833, doi:10.5194/angeo-20-823-2002, 2002.

Karpova, N. V., Petrova, L. N., and Shved, G. M.: Atmospheric and Earth-surface oscillations with steady frequencies in the 0.7$1.5 \mathrm{~h}$ and $2.5-5.0 \mathrm{~h}$ period range, Izvestiya, Atmos. Ocean. Phys., Engl. Transl., 40, 10-20, 2004.

Kozhevnikova, E. G., Orlov, E. G., and Lin'kov, E. M.: Pressure variations as a noise for long-period observations, in: Problems of geophysics, 28, edited by: Molochnov, G. V. and Semenov, A. S., Leningrad University Press, Leningrad, 229-233, 1980.

Lin'kov, E. M.: Seismic phenomena, Leningrad University Press, Leningrad, 1987.

Lin'kov, E. M., Petrova, L. N., and Zuroshvili, D. D.: Seismogravitational oscillations of the Earth and associated disturbances of the atmosphere, Trans. USSR Acad. Sci.: Earth Sci., Engl. Transl., 306, 13-16, 1991.

Livneh, D. J., Seker, I., Djuth, E. T., and Mathews, J. D.: Continuous quasiperiodic thermosphere waves over Arecibo, J. Geophys. Res., 112, A07313, doi:10.1029/2006JA012225, 2007.

Lognonné, P., Clévédé, E., and Kanamori, H.: Computation of seismograms and atmospheric oscillations by normal-mode summation for a spherical earth model with realistic atmosphere, Geophys. J. Int., 135, 388-406, 1998.

Madden, R. A.: Large-scale, free Rossby waves in the atmosphere - an update, Tellus, 59A, 571-590, 2007.

Malinina, T. I.: Seiches of the lake Ladoga, in: Hydrologic regime and water balance of the lake Ladoga, Transactions of Laboratory for Limnology, 20, Leningrad University Press, Leningrad, 229246, 1966.

Merriam, J. B.: Atmospheric pressure and gravity, Geophys. J. Int., 109, 488-500, 1992.

Müller, T. and Zürn, W.: Observation of gravity changes during the passage of cold fronts, J. Geophys., 53, 155-162, 1983.

Nawa, K., Suda, N., Fukao, Y., Sato, T., Aoyama, Y., and Shibuya, K.: Incessant excitation of the Earth's free oscillations, Earth Planets Space, 50, 3-8, 1998; Reply, ibid., 50, 887-892, 1998.

Pancheva, D., Merzlyakov, E., Mitchell, N. J., Portnyagin, Yu., Manson, A. H., Jacobi, Ch., Meek, C. E., Luo, Y., Clark, R. R., Hocking, W. K., MacDougall, J., Muller, H. G., Kürschner, D., Jones, G. O. L., Vincent, R. A., Reid, I. M., Singer, W., Igarashi, K., Fraser, G. I., Fahrutdinova, A. N., Stepanov, A. M., Poole, L. M. G., Malinga, S. B., Kashcheyev, B. L., and Oleynikov, A. N.: Global-scale tidal variability during the PSMOS campaign of June-August 1999: interaction with planetary waves, J. Atmos. Solar-Terr. Phys., 64, 1865-1896, 2002.

Petrova, L. N.: Seismogravitational oscillations of the Earth from observations by spaced vertical pendulums in Eurasia, Izvestiya, Phys. Solid Earth, Engl. Transl., 38, 325-336, 2002.
Petrova, L. N. and Lyubimtsev, D. V.: Global nature of the seismogravitational oscillations of the Earth, Izvestiya, Phys. Solid Earth, Engl. Transl., 42, 114-123, 2006.

Petrova, L. N. and Shved, G. M.: Revealing short-period global oscillations of the atmosphere from seismic observations, Izvestiya, Atmos. Ocean. Phys., Engl. Transl., 36, 64-67, 2000.

Petrova, L. N., Osypov, K. S., Savel'ev, D. D., and Shved, G. M.: Forcing atmospheric oscillations by long-period seismic oscillations: a case study, J. Atmos. Terr. Phys., 58, 1317-1322, 1996.

Pokrovskaya, I. V. and Sharkov, E. A.: Tropical cyclones and tropical disturbances of the World Ocean: Chronology and evolution. Version 2.1 (1983-2000), Poligraph servis, Moscow, 2001.

Press, W. H., Teukolsky, S. A., Vetterling, W. T., and Flannery, B. P.: Numerical recipes in FORTRAN 77: The art of scientific computing, Cambridge Univ. Press, New York, 569-577, 1992.

Rosat, S., Sato, T., Imanishi, Y., Hinderer, J., Tamura, Y., McQueen, H., and Ohashi, M.: High-resolution analysis of the gravest seismic normal modes after the $2004 \mathrm{M}_{w}=9$ Sumatra earthquake using superconducting gravimeter data, Geophys. Res. Lett., 32, L13304, doi:10.1029/2005GL023128, 2005.

Savarensky, E. F. and Kirnos, O. P.: An introduction to seismology and seismometry, GITTL, Moscow, 1955.

Scargle, J. D.: Studies in astronomical time series analysis. II. Statistical aspects of spectral analysis of unevenly space data, Astrophys. J., 263, 835-853, 1982.

Shved, G. M., Petrova, L. N., and Polyakova, O. S.: Penetration of the Earth's free oscillations at 54 minute period into the atmosphere, Ann. Geophys., 18, 566-572, doi:10.1007/s00585-0000566-0, 2000.

Sidorenkov, N. S.: Atmospheric processes and Earth's rotation, Gidrometeoizdat, St. Petersburg, 2002.

Smylie, D. E., Hinderer, J., Richter, B., and Ducarme, B.: The product spectra of gravity and barometric pressure in Europe, Phys. Earth Planet. Inter., 80, 135-157, 1993.

Sorrells, G. G.: A preliminary investigation into the relationship between long-period seismic noise and local fluctuations in the atmospheric pressure field, Geophys. J. R. Astr. Soc., 26, 71-82, 1971.

Spratt, R. S.: Modelling the effect of atmospheric pressure variations on gravity, Geophys. J. R. Astr. Soc., 71, 173-186, 1982.

Walterscheid, R. L. and Sivjee, G. G.: Very high frequency tides observed in the airglow over Eureka $\left(80^{\circ}\right)$, Geophys. Res. Lett., 23, 3651-3654, 1996.

Walterscheid, R. L. and Sivjee, G. G.: Zonally symmetric oscillations observed in the airglow from South Pole station, J. Geophys. Res. (A), 106, 3645-3654, 2001.

Warburton, R. J. and Goodkind, J. M.: The influence of barometricpressure variations on gravity, Geophys. J. R. Astr. Soc., 48, 281292, 1977.

Weatherford, C. L. and Gray, W. M.: Typhoon structure as revealed by aircraft reconnaissance. Part I: Data analysis and climatology, Mon. Weather Rev., 116, 1032-1043, 1988.

Wielandt, E.: Seismometry, in: International handbook of earthquake and engineering seismology, 81A, edited by: Lee, W. H. K., Kanamori, H., Jennings, P. C., and Kisslinger, C., Academic Press, London and San Diego, 283-304, 2002.

Wu, Q., Killeen, T. L., McEwen, D., Solomon, S. C., Guo, W., Sivjee, G. G., and Reeves, J. M.: Observation of the mesospheric and lower thermospheric 10-hour wave in 
the northern polar region, J. Geophys. Res., 107(A6), 1082, doi:10.1029/2001JA000192, 2002.
Zürn, W. and Widmer, R.: On noise reduction in vertical seismic records below $2 \mathrm{mHz}$ using local barometric pressure, Geophys. Res. Lett., 22, 3537-3540, 1995. 